\title{
Early Cretaceous (Neocomian-Cenomanian) Palynomorphs
}

\author{
B. THUSU \& J. G. L. A. VAN DER EEM \\ Exploration Laboratory, Arabian Gulf Oil Company, PO Box 263, Benghazi, Libya, S.P.L.A.J.
}

\section{INTRODUCTION}

This study is primarily concerned with the Neocomian to Aptian palynomorphs recorded in selected exploration wells (See Fig. 9). In order to document a complete Early Cretaceous microfloral succession in the studied wells, a reconnaissance of Aptian to Early Cenomanian palynomorphs was also undertaken. Details of the results from this younger interval appear in a later section.

Palynomorph assemblages vary in preservation and character. To the north, sandstone, siltstone and shale deposited in shallow-marine environments, contain wellpreserved assemblages of dinoflagellate cysts, pollen and spores which can be used for stage-level age determination. A majority of the samples analysed, however, contain moderate numbers of dinoflagellate cysts, but fewer miospores. The abundance of land derived detritus indicates the relatively close proximity of the shoreline. In the central and southern parts of the study area, sandstone and siltstone that are deposited in nonmarine, fluvial, lacustrine or lagoonal environments show a general paucity of well-preserved palynofloras. Miospores of stratigraphic value are generally rare or absent although the majority of the samples are dominated by land derived detritus.

\section{PALYNOMORPH SUCCESSION}

The majority of the wells from northern Cyrenaica show a hiatus at the Jurassic Cretaceous boundary. Late Neocomian or Aptian sediments occur immediately above Middle or early Late Jurassic sediments. Well preserved Neocomian palynomorphs were recorded in wells A1-36, B1-36, Bla-18 and A1-45. The stratigraphical ranges of palynomorphs presented on the plate explanations are local ranges and are based on the studied intervals only. A preliminary palynological zonation of Late Jurassic (Late Kimmeridgian) to Late Cretaceous (Cenomanian) is presented in Fig. 9. This zonation is based on samples dated independently on microfaunal assemblages. Further refinement of this zonation is currently in progress.

Neocomian palynomorph assemblages are moderately diverse in composition containig both dinoflagellate cysts and miospores. The dinoflagellate cysts $M$ uderongia simplex, $M$. staurota, Phoberocysta neocomica and several species of Canningia and Cyclonephelium make their first appearance during this interval. Miospores do not dominate the assemblages although there are large quantities of terrestrial palynodebris in the majority of the samples studied. Bisaccate pollen are generally absent. Rare but consistently present miospores include Trilobosporites cf. bernissartensis, Matonisporites crassiangulatus, Equitriradites spinulosus, Crybelosporites cf. striatus (similar to $C$. striatus but larger in size), and several species of Pilosisporites, Concavisporites, Classopollis, Concavissimisporites, Cicatricosisporites and inaperturate pollen (Araucariacites, Inaperturopollenites). Callialasporites and Exesipollenites occur sporadically. Microfloral subdivision within the Neocomian must await the completion of the study of additional samples.

Late Neocomian to Barremian palynomorphs are well-preserved but occur only in small numbers. The dinoflagellate cysts Aptea anaphrissa, Gardodinium eisenacki, Coronifera oceanica and several species of Oligosphaeridium, in association with the miospores Dicheiropollis etruscus, Stellatopollis cf. barghoornii. Appendicisporites spp., Retimonocolpites spp. and "Inaperturopollenites" crisopolensis show their earliest appearance in this interval. Some species from the Early Neocomian continue as infrequent or rare components, these include Pareodinia ceratophora, Prolixosphaeridium granulosum, Canningia spp., Cyclonephelium spp., Cicatricosisporites spp., Crybelosporites cf. striatus, Concavisporites spp., Classopollis spp., and inaperturate pollen.

Aptian dinoflagellate cyst assemblages contain abundant representatives of Subtilisphaera senegalensis and Subtilisphaera sp. together with rare Pseudoceratium regium and Cribroperidinium edwardsii. Miospores from this interval contain the first appearance of A fropollis spp., Reyrea polymorpha, Retitricolpites spp., Psilatricolpites spp. and Eucomiidites sp. Classopollis is represented by abundant species of less than 30 microns. A number of species which disappear during this interval include "Inaperturopollenites" crisopolensis, Stellatopollis cf. barghoornii, Callialasporites spp., and Appendicisporites spp. Ephedripites spp., present in the Neocomian is more frequently encountered during the late Early Cretaceous. Inaperturate pollen, Crybelosporites, Cicatricosisporites, Concavisporites and Pilosisporites continue as infrequent members of the assemblages.

Albian-Cenomanian palynomorph assemblages contain a number of distinctive dinoflagellate cysts including Xenascus ceratioides, Cyclonephelium vanno- 
phorum and Florentina spp. Miospore assemblages from this interval are distinguished by the earliest appearance during the Albian of Elaterosporites klaszii and Elaterocolpites castelainii together with Sofrepites legouxae, tricolporate and triporate angiosperm pollen. Species of Classopollis less than 30 microns are replaced by larger forms of which $C$. brasiliensis is a good stratigraphic marker. Species of Afropollis and of the Elaterosporites group disappear in the Early Cenomanian but Cicatricosisporites, Crybelosporites, Retimonocolpites, Tricolpites and inaperturate pollen continue to occur.

\section{SARIR (NUBIAN) SANDSTONE}

Sediments from within this unit, in the central and southern parts of the project area, where it was deposited in fluvial, lacustrine or lagoonal environments show a general paucity of well preserved palynomorphs. Miospores of stratigraphical value are generally rare. Taxa frequently present include Cerebropollenites mesozoicus, Concavisporites spp., Classopollis spp., Araucariacites $\mathrm{sp}$. and rarely Alisporites $\mathrm{sp}$. These miospores are not age diagnostic but suggest a Late Jurassic-Early Cretaceous age for the Sarir or Nubian sandstone. Samples from a limited number of wells have yielded microfloras which can be dated confidently as Neocomian-
Barremian, by the presence of Dicheiropollis etruscus, Trilobosporites sp. and Cupressacites oxycedroides. In one well, the rare occurence of Psilatricolporites sp. and Stellatopollis barghoornii indicates an Aptian-Albian age.

\section{REFERENCES}

Doyle, J. A., Jardiné, S. \& Doerenkamp, A. 1982. A fropollis, a new genus of early angiosperm pollen, with notes on the Cretaceous palynostratigraphy and palaeoenvironments of northern Gondwana. Bull. Centres Rech. Explor. - Prod. Elf-Aquitaine, 6 (1), 39-117.

Herngreen, G. F. W. \& Chlonova, A. F. 1981. Cretaceous microfloral provinces. Pollen et Spores, 23 (3-4), 441-555.

Jansonius, J. \& Hills, L. V. 1976. Genera file of fossil spores (plus supplements).Spec. Publ. -Dept. Geol. Univ. Calgary.

Lentin, J. K. \& Williams, G. L. 1981. Fossil dinoflagellates: Index to genera and species, 1981 edition. Report Ser. Bedford Instit. Oceanogr., B1-R-81-12, 1-345.

Singh, C. 1971. Lower Cretaceous microfloras of the Peace River area, northwestern Alberta. Bull. Res. Counc. Alberta, 28 (1-2), $542 \mathrm{pp}$.

Urban, L. L., Moore, L. V. \& Allen, M. L. 1976. Palynology, thermal alteration and source rock potential of three wells from Alain area, Western Desert, Egypt. Fifth EGCP Exploration Seminar, November 1976, $32 \mathrm{pp}$. 


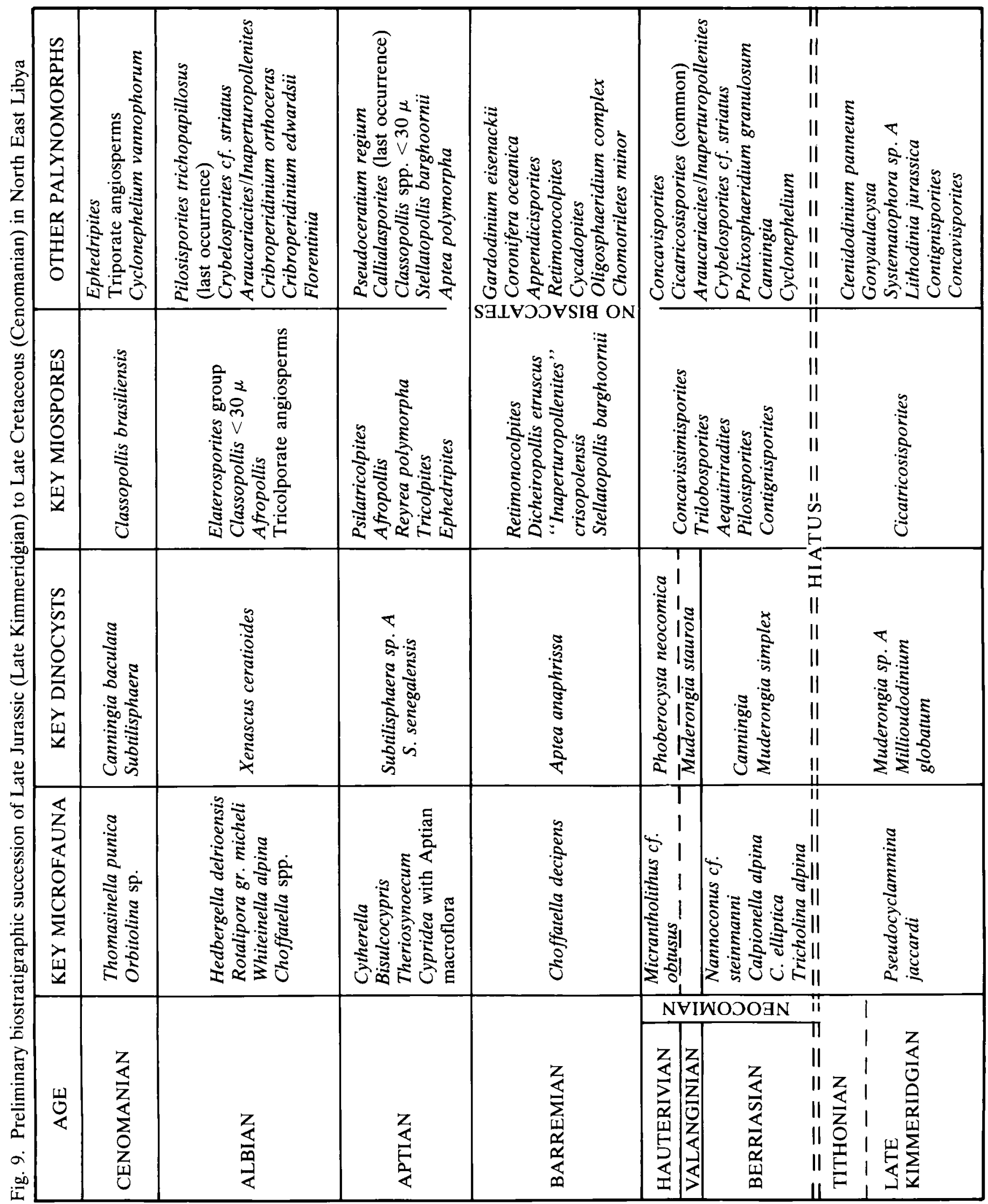




\section{Explanation of Plate 53}

All figures are $\times 500$

Figs. 1, 3. Canningia reticulata Cookson \& Eisenack, 1960 emend. Below 1981 1. A1-45, 12100-12250ft., Slide 3 , R44/0, AGC 586. 3. A1-NC92, 5880-5890ft., Slide 3, N44/0, AGC 587. (Neocomian-Albian).

Fig. 2. Cyclonephelium distinctum Deflandre \& Cookson, 1955. A1-36, 10607-10617ft., Slide 1, L31/0, AGC 588. (Neocomian-Albian).

Fig. 4. Muderongia simplex Alberti, 1961. B1-36, 10915-11010ft., Slide 2, M29/2, AGC 589. (Early Neocomian).

Figs. 5, 6. Phoberocysta neocomica subsp. neocomica (Gocht, 1957) Millioud, 1969. 5. B1-36, 10734-10738ft., Slide 2, R43/3, AGC 590. 6. A1-36, 10607-10617ft., Slide 3, N45/1, AGC 591. (Neocomian).

Figs. 7, 8. Muderongia cf. staurota Sarjeant, 1966. 7. A1-45, 9710-9900ft., Slide 3, N40/0, AGC 592. 8. A1-45, 10110-10300ft., Slide 2, N38/4, AGC 593. (Neocomian).

Fig. 9. Florentinia sp. A. A1-45, 10110-10300ft., Slide 2, Q28/0, AGC 594. (Neocomian).

Fig. 10. Phoberocysta neocomica subsp. circulata (Gocht, 1957) Lentin \& Williams, 1973. R3-82, $3658 \mathrm{~m}$., Slide 1, L41/1, AGC 595. (Neocomian).

Fig. 11. Odontochitina operculata (Wetzel, 1933), Deflandre \& Cookson, 1955. A1-45, 9710-9900ft., Slide 3, J33/0, AGC 596. (Neocomian). 
B. Thusu \& J. G. L. A. Van Der Eem
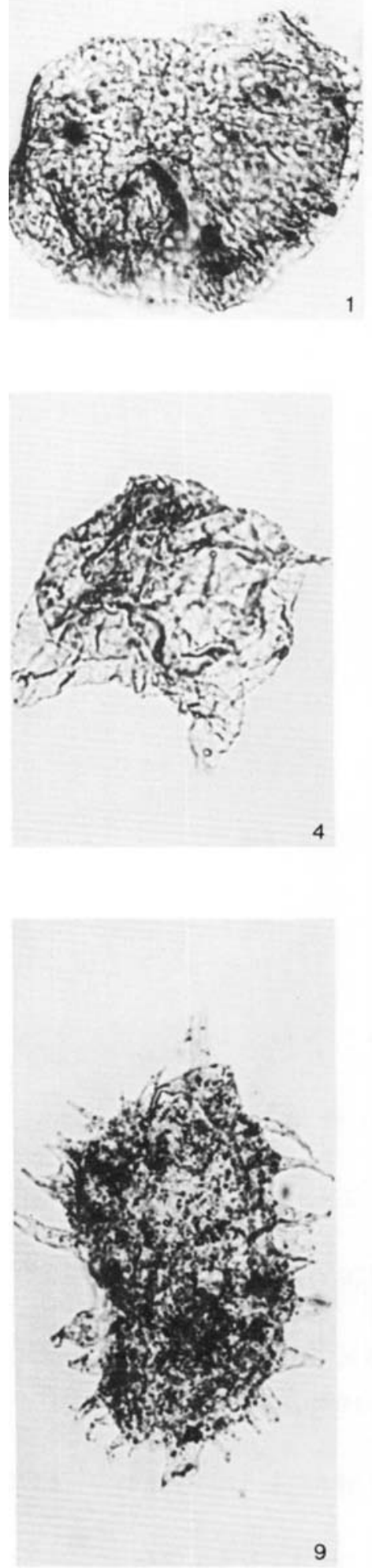
Neocomian Dinoflagellate Cysts

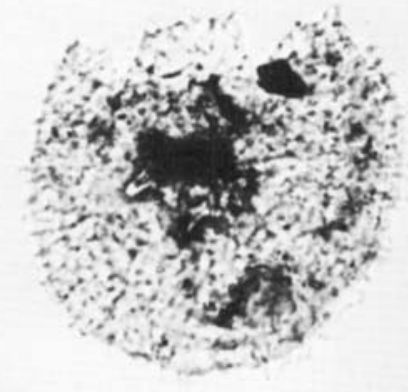

Plate 53
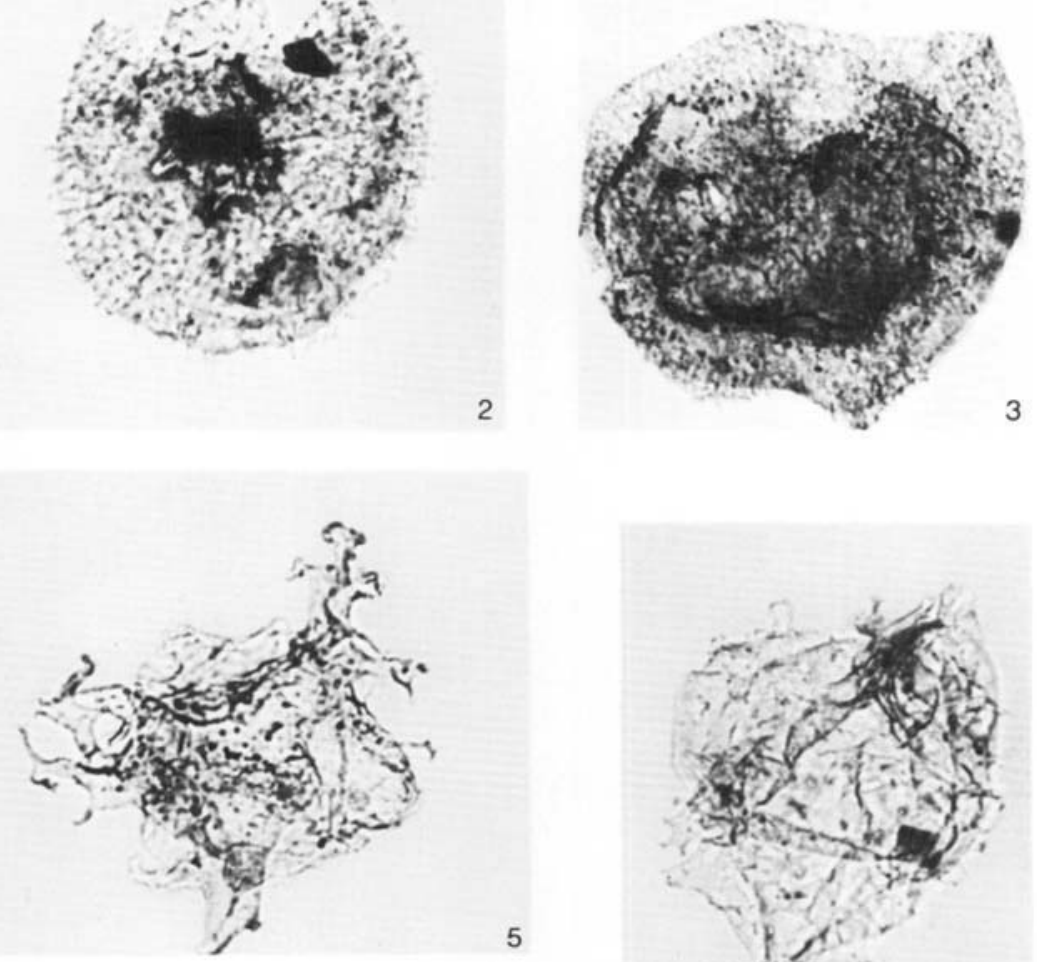

5
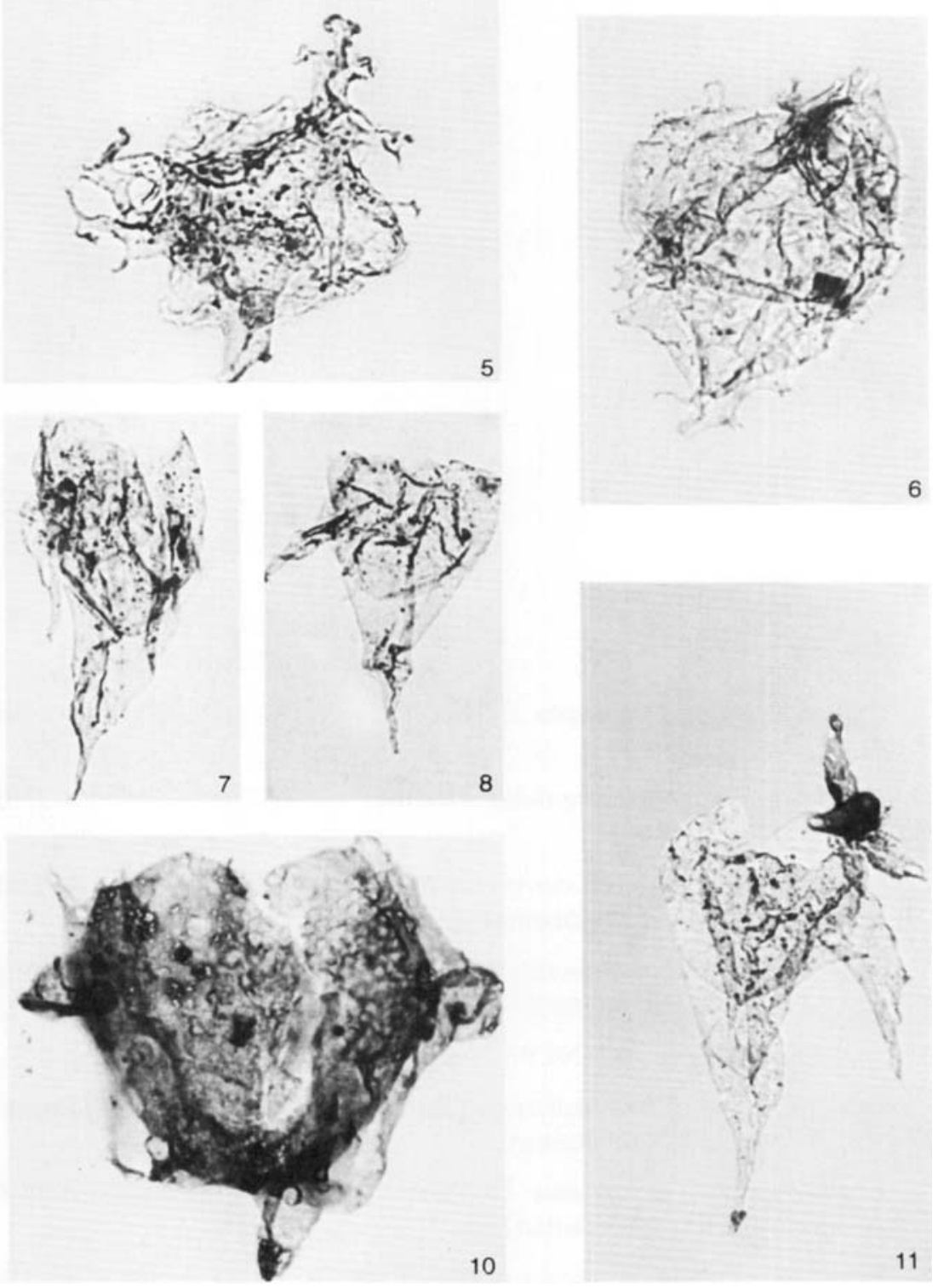


\section{Explanation of Plate 54}

All figures are $\times 500$

Fig. 1. Concavisporites jurienensis Balme, 1957. A1-36, 7870-7880ft., Slide 2, Y39/1, AGC 597. (NeocomianAlbian).

Fig. 2. Matonisporites crassiangulatus (Balme, 1957) Dettmann, 1963. Bla-18, 7900-8000ft., Slide 4, N44/3, AGC 598. (Neocomian).

Fig. 2. Concavissimisporites cf. variverrucatus (Couper, 1958) Brenner, 1963. GGG1-59, Core 11, 11382ft., Slide 2B, F49/0, AGC 599. (Neocomian).

Fig. 4. Concavissimisporites punctatus (Delcourt \& Sprumont, 1955) Brenner, 1963. A1-36, 7014-7066ft., Slide 2, F34/1, AGC 600. (Neocomian-Albian).

Fig. 5. Concavisporites sp. A1-NC92, 6630-6640ft., Slide 3, Y32/4-K32/3, AGC 601. (Neocomian-Cenomanian).

Fig. 6. Trilobosporites cf. berrissartensis (Delcourt \& Sprumont, 1955) Potonié, 1956. Bla-18, 8500-8600ft., Slide 4, U35/4, AGC 602. (Neocomian).

Fig. 7. Crybelosporites cf. striatus Dettmann, 1963. Bla-18, 5600-5700ft., Slide 4, U29/1-T29/3, AGC 603. (Neocomian-Early Cenomanian).

Fig. 8. Impardecispora apiverrucata (Couper, 1958) Venkatachala et al, 1968. Bla-18, 6900-7000ft., P40/4-41/3, AGC 604. (Neocomian). 

Neocomian Miospores
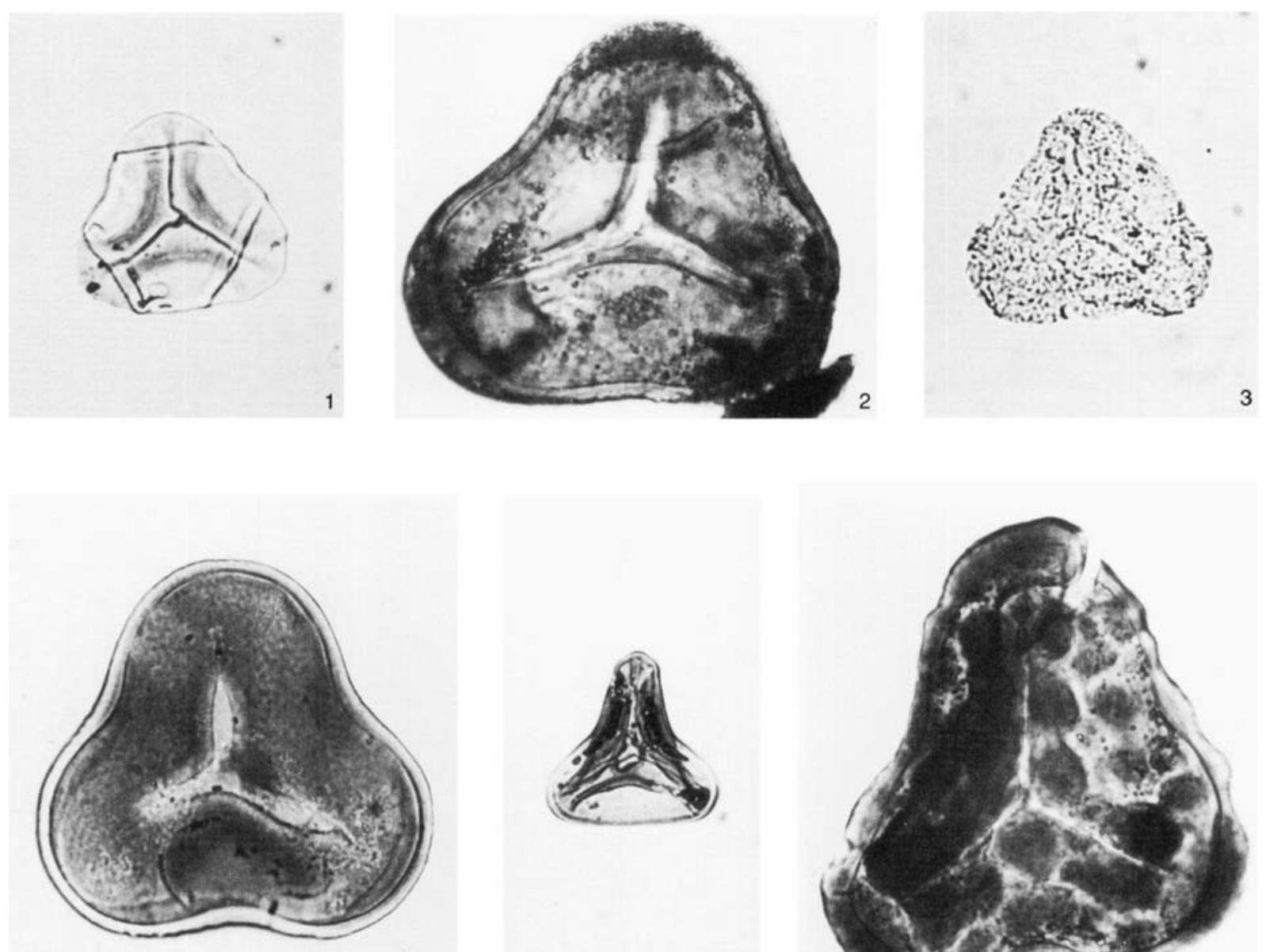

4
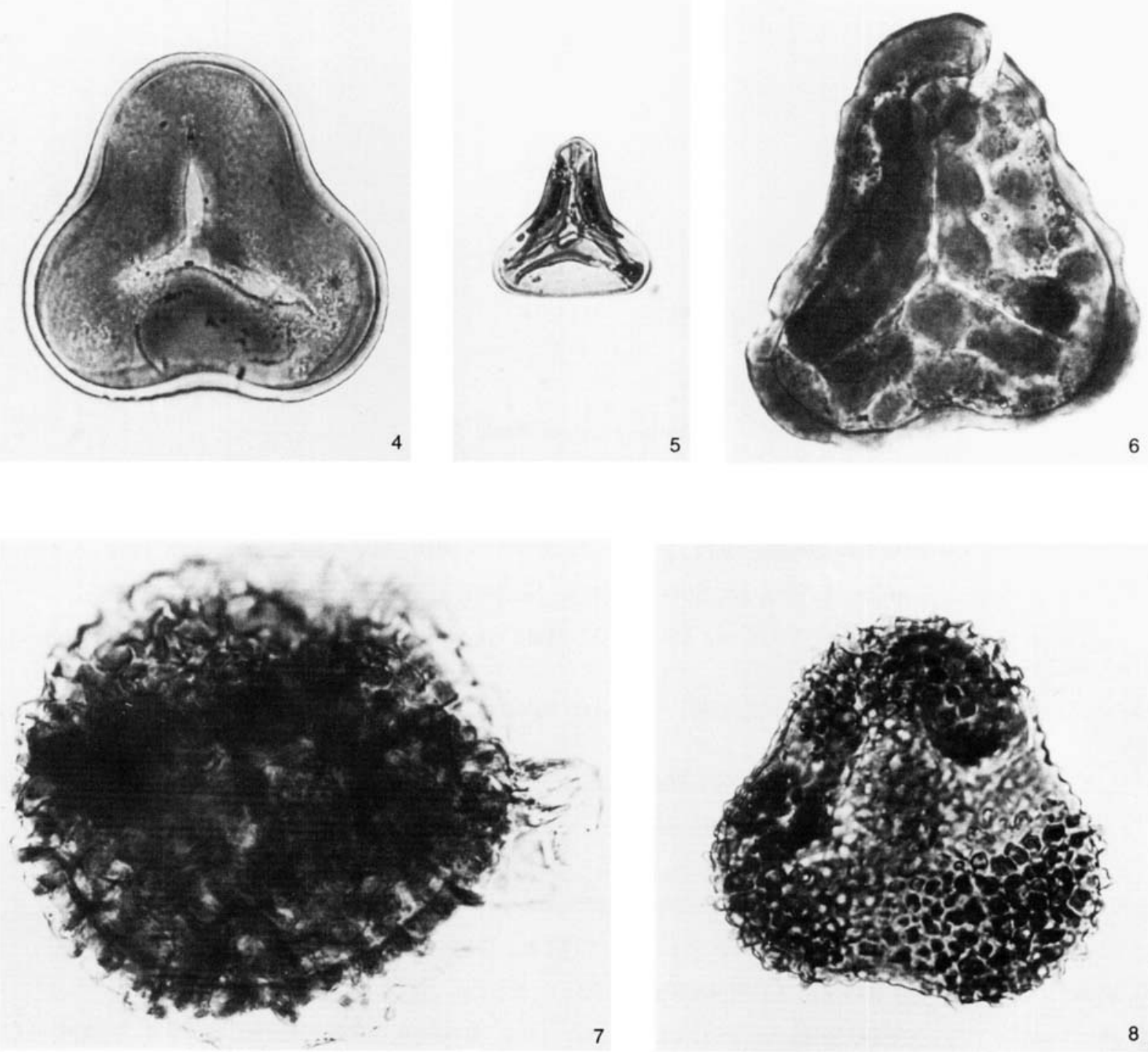


\section{Explanation of Plate 55}

All figures are $\times 500$

Fig. 1. Cicatricosisporites sp. A. Bla-18, 8240-8300ft., Slide 4, N36/4, AGC 605. (Neocomian).

Fig. 2. Tigrisporites cf. reticulatus Singh, 1971. B1-36, 10610-10710ft., Slide 3, K34/0, AGC 606. (Neocomian).

Fig. 3. Genus and species indet. 1. B1a-18, 3900-4000ft., Slide 4, Q26/1, AGC 607. (Neocomian).

Fig. 4. Gen. and spec. indet. 2. A1-36, Core 10, 10607-10617ft., Slide 1 unox., U27/3, AGC 608. (NeocomianBarremian).

Fig. 5. Pilosisporites sp. 2 sensu Bebout, 1981. B1a-18, 8240-8300ft., Slide 4, T28/1, AGC 609. (NeocomianBarremian).

Fig. 6. Inaperturopollenites sp. B1 a-18, 3700-3800ft., Slide 4, Y46/1, AGC 610. (Neocomian-Cenomanian).

Fig. 7. Cicatricosisporites sp. B. B1a-18, 3700-3800ft., Slide 4, N39/2, AGC 611. (Neocomian-Aptian).

Fig. 8. Aequitriradites spinulosus (Cookson \& Dettman, 1958) Cookson \& Dettman, 1961. B1a-18, 7400-7500ft., Slide 4, L35/0-35/4, AGC 612. (Neocomian-Barremian).

Fig. 9. Verrucosisporites sp. B1-36, Core 24, 11173-11181ft., Slide 2, K45/3, AGC 613. (Neocomian).

Fig. 10. Contignisporites sp. B1a-18, 4700-4800ft., Slide 4, Y25/0, AGC 614. (Neocomian).

Fig. 11. Araucariacites australis Cookson, 1947 ex Couper, 1953. B1a-18, 5400-5500ft., Slide 4, V37/4, AGC 615. (Neocomian-Cenomanian). 
B. Thusu \& J. G. L. A. Van Der Eem Neocomian Miospores
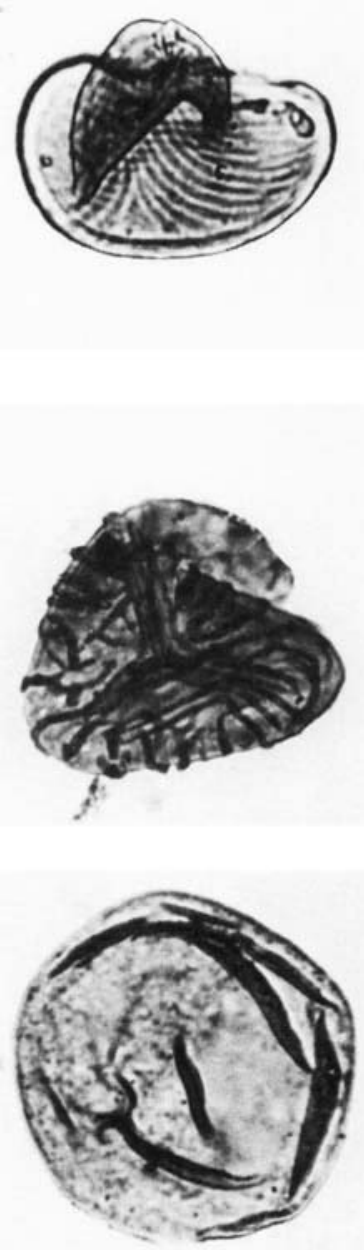

4
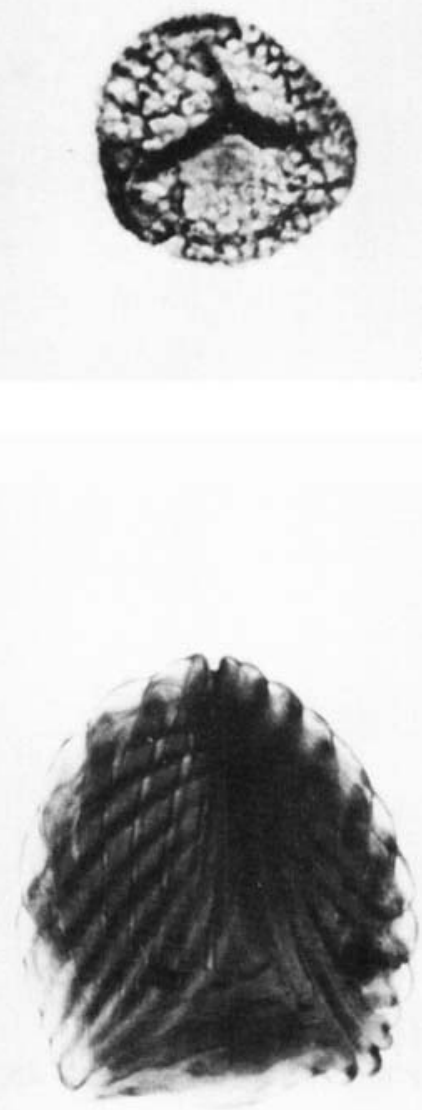

2

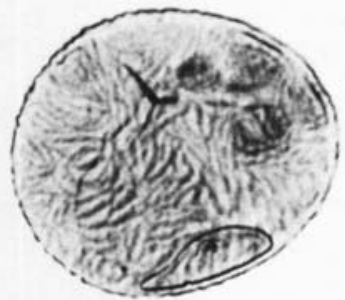

3

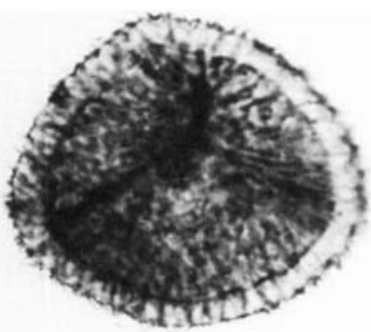

5

7
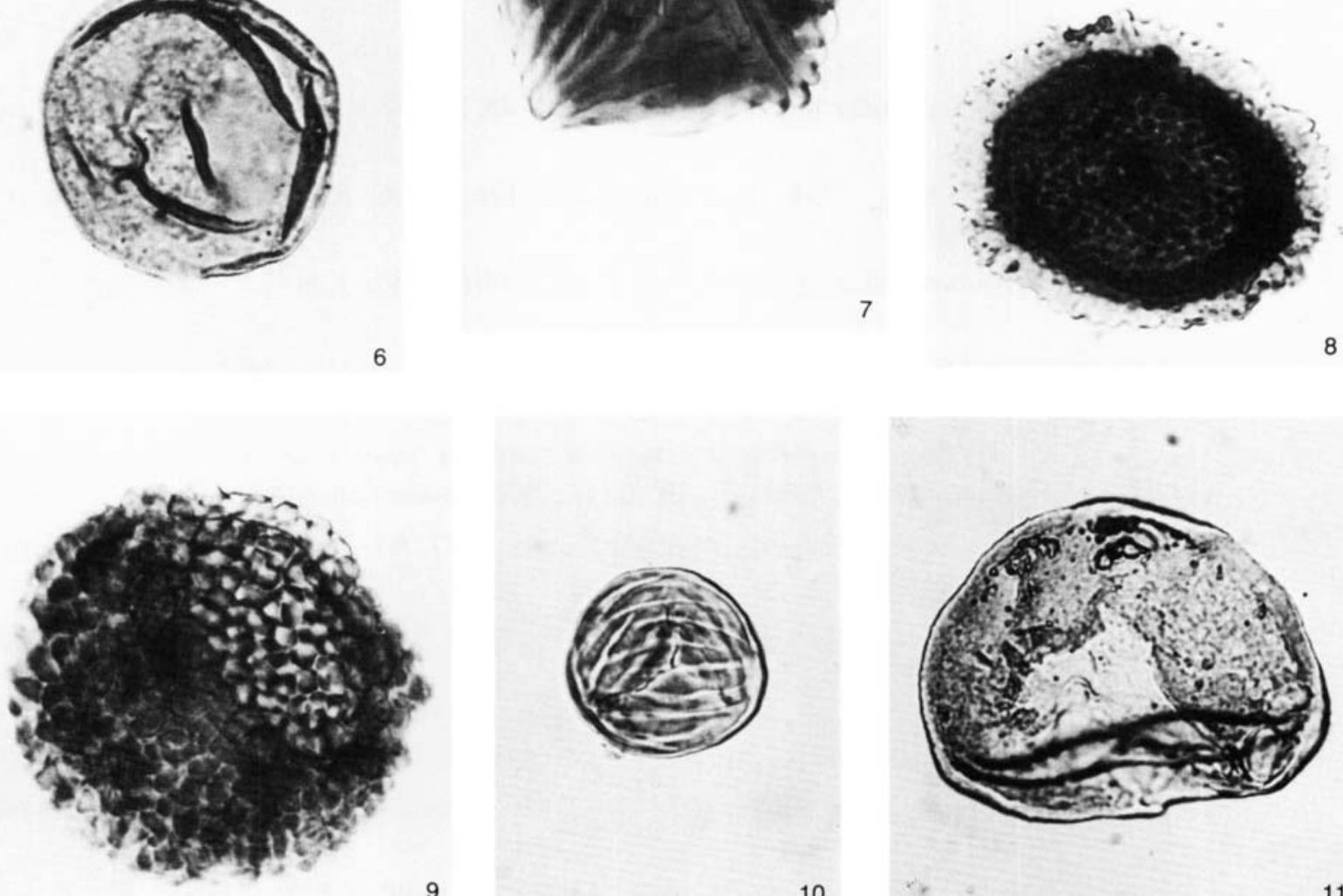

Plate 55 


\section{Explanation of Plate 56}

All figures are $\times 500$

Fig. 1. Cyclonephelium distinctum Deflandre \& Cookson, 1955. A1-45, 10510-10700ft., Slide 3, V32/1, AGC 616. (Neocomian-Albian).

Fig. 2. Spiniferites ramosus (Ehrenberg, 1938) Loeblich \& Loeblich, 1966. R3-82, 3658m., Slide 1, Q32/0, AGC 617. (Neocomian-Albian).

Fig. 3. Cyclonephelium vannophorum Davey, 1969. A1-45, 9710-9900ft., Slide 3, H47/2, AGC 618. (NeocomianCenomanian).

Fig. 4. Hystrichodinium furcatum Alberti, 1961. A1-45, 9710-9900 ft., Slide 2, N41/1, AGC 619. (Late NeocomianBarremian).

Figs. 5, 10. Oligosphaeridium perforatum (Gocht, 1959) Davey \& Williams, 1969. 5. R3-82, 4415m., Slide 1, S43/4, AGC 620. 10. R3-82, 3784 m., Slide 1, U32/1, AGC 621. (Late Neocomian-Albian).

Fig. 6. Glabridinium apatelum (Cookson \& Eisenack, 1960) Brideaux, 1977. A1-36, 10607-10617ft., Slide 1, J28/4, AGC 622. (Neocomian-Barremian).

Fig. 7. Coronifera oceanica Cookson \& Eisenack, 1958, emend. May, 1980. A1-NC 92, 6200-6210ft., Slide 4, S37/2, AGC 623. (Late Neocomian-Albian).

Figs. 8, 11. Oligosphaeridium complex (White, 1842) Davey \& Williams, 1966. 8. A1-45, 10920-11100ft., Slide 2, T33/1, AGC 624. 11. A1-45, 9710-9900ft., Slide 3, O39/1, AGC 625. (Late Neocomian-Albian).

Fig. 9. Gardodinium eisenackii Alberti, 1961. B1-36, 10734-10738ft., Slide 3, E34/0, AGC 626. (Late NeocomianBarremian).

Fig. 12. Aptea anaphrissa (Sarjeant, 1966), Benedek, 1972. A1-NC 92, 6100-6110ft., Slide 5, P26/0, AGC 627. (Barremian). 
B. Thusu \& J. G. L. A. Van Der Eem

Plate 56

Late Neocomian-Barremian Dinoflagellate Cysts

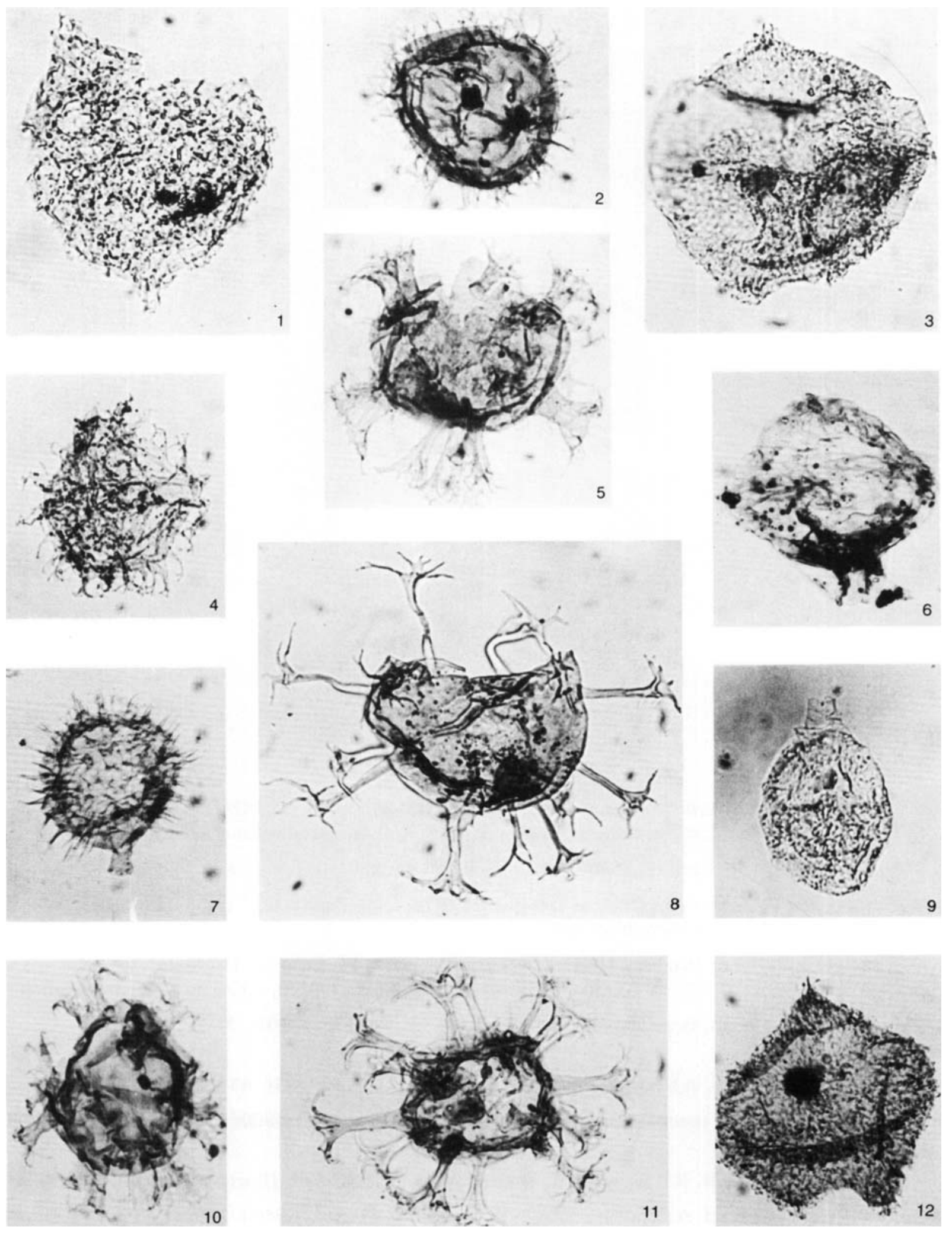




\section{Explanation of Plate 57}

All figures are $\times 500$

Figs. 1, 3. Dicheiropollis etruscus Trevisian, 1972. 1. GGG1-59, Core 11, $11382 \mathrm{ft}$., Slide 4, H25/0, AGC 628. 3. GGG1-59, Core 11, $11382 \mathrm{ft}$., Slide 4, F27/0, AGC 629. (Late Neocomian-Barremian).

Fig. 2. Cycadopites sp. A1-36, Core 10, 10607-10617ft., Slide 1 unox., N30/0, AGC 630. (Neocomian-Cenomanian).

Figs. 4, 7. Stellatopollis cf. barghoornii Doyle in Doyle et al., 1976. 4. proximal side, 7. distal side, L1-82, 3695-3707ft., Slide 4, Q35/3, AGC 631. (Barremian-Aptian).

Figs. 5, 9. Chomotriletes minor (Kedves, 1961), Pocock, 1970. 5. B1-36, Core 22, 10734-10738ft., Slide 3, R26/427/3 - S26/2-27/1, AGC 632. 9. B1-36, 10610-10710ft., Slide 2, O40/0, AGC 633. (Neocomian-Albian).

Fig. 6. “Inaperturopollenites" crisopolensis Regali et al, 1975. G1-2, 7170-7210ft., Slide 2, O30/0, AGC 634. (Late Barremian-Aptian).

Fig. 8. Retimonocolpites sp. A. A1-NC92, 6200-6210ft., Slide 4, M26/0, AGC 635. (Barremian-Cenomanian).

Fig. 10. Appendicisporites cf. potomacensis Brenner, 1963. B1a-18, 4700-4800ft., Slide 4, O45/1, AGC 636. (Barremian-Aptian).

Fig. 11. Retimonocolpites sp. B. R3-82, 4209m., Slide 1, W35/3, AGC 637. (Barremian-Cenomanian).

Fig. 12. Appendicisporites sp. cf. A. bifurcatus Singh, 1964. R3-82, 4118 m., Slide 1, N25/2, AGC 638. (BarremianAptian). 


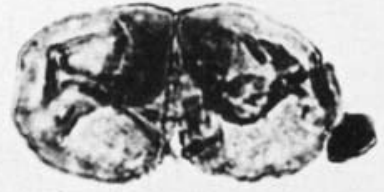

1

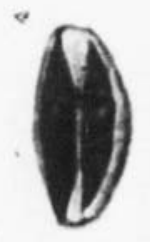

2
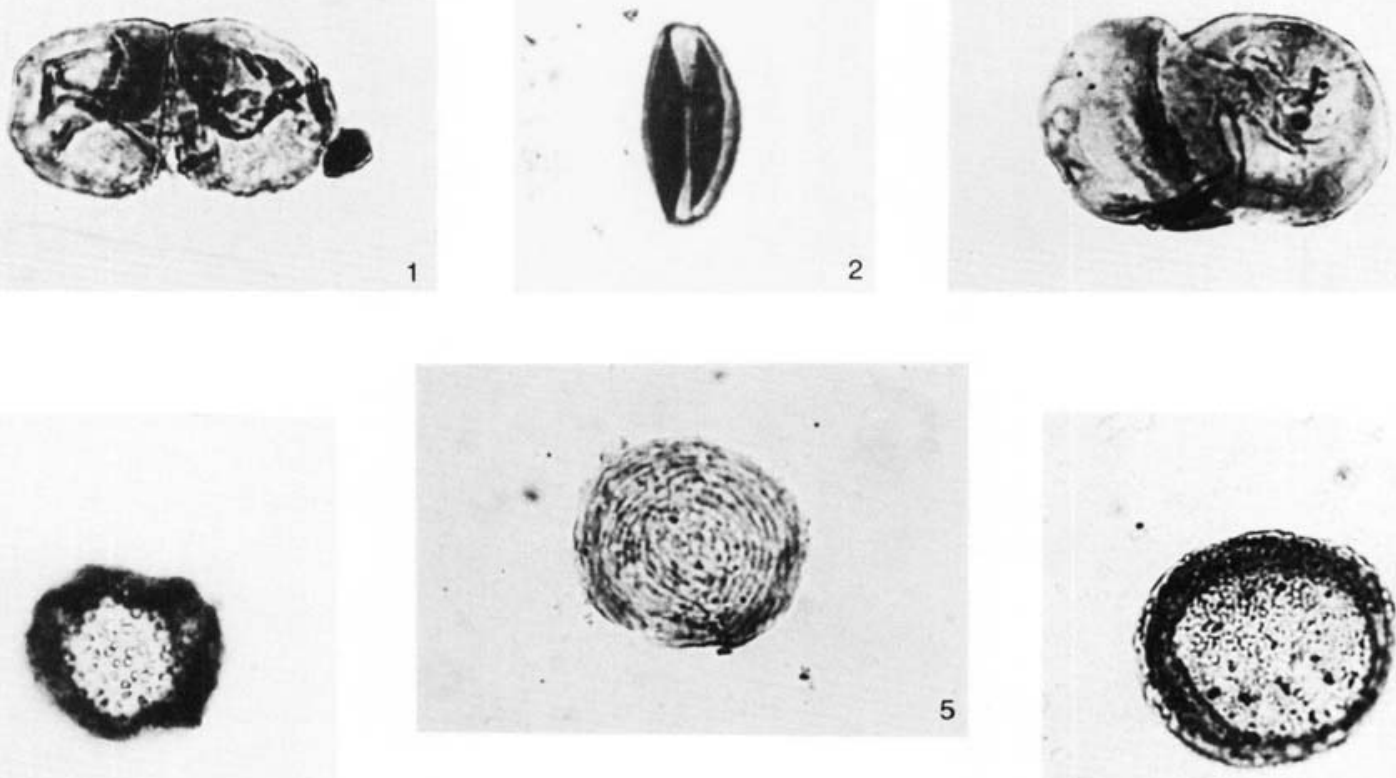

4

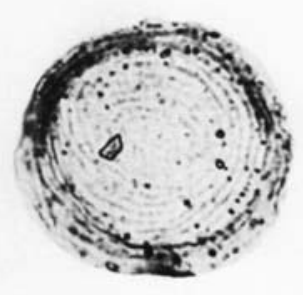

7

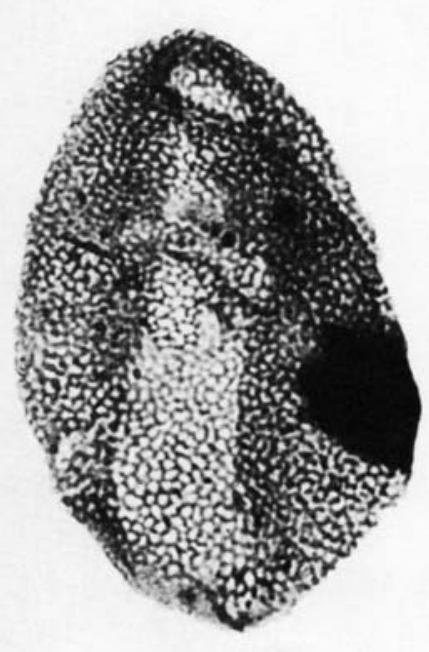

8
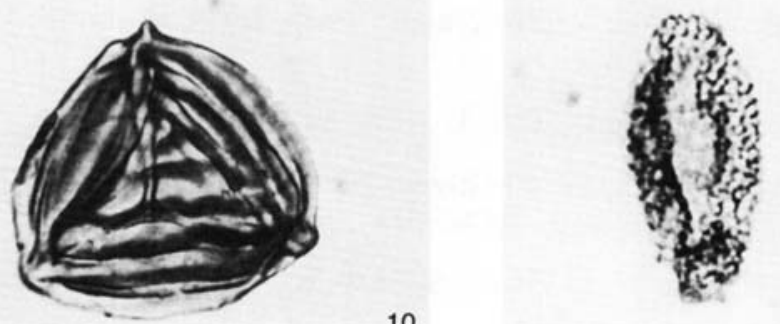

11

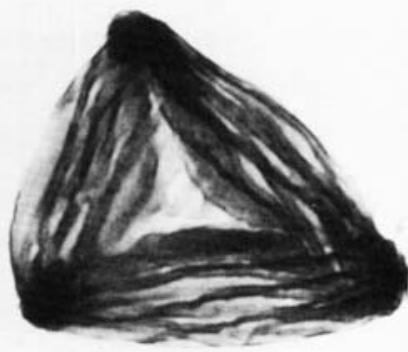




\section{Explanation of Plate 58}

All figures are $\times 500$

Figs. 1, 2. Subtilisphaera pirnaensis (Alberti, 1959) Jain \& Millepied, 1973. 1. A1-45, 10510-10700ft., Slide 3, U28/2, AGC 639. 2. A1-45, 12100-12250ft., Slide 3, N38/3, AGC 640. (Aptian-Albian).

Fig. 3. Millioudodinium episomum (Sarjeant, 1966) Stover \& Evitt, 1978. A1-45, $11110-11300 \mathrm{ft}$., Slide 3, M47/1, AGC 641. (Aptian).

Fig. 4. Subtilisphaera senegalensis Jain \& Millepied, 1973. A1-45, 10110-10300ft., Slide 2, N34/0, AGC 642. (Aptian-Albian).

Fig. 5. Spiniferites ramosus (Ehrenberg, 1938) Loeblich \& Loeblich, 1966. A1-45, 12100-12250ft., Slide 3, O30/0, AGC 643. (Neocomian-Albian).

Fig. 6. Cribroperidinium cf. edwardsii (Cookson \& Eisenack, 1958) Davey, 1969. B1a-18, 4400-4500ft., Slide 4, K40/1, AGC 644. (Aptian-Albian).

Fig. 7. ?Lagenorhytis sp. A. A1-NC 92, 6640-6650ft., Slide 6, K36/3, AGC 645. (? Aptian).

Figs. 8, 9. Cribroperidinium edwardsii (Cookson \& Eisenack, 1958) Davey, 1969. 8. A1-36, 7870-7880ft., Slide 4, N34/0, AGC 646. 9. A1-45, 9910-10100 ft., Slide 2, U40/2, AGC 647. (Aptian-Albian).

Fig. 10. Pseudoceratium regium Singh, 1971. A1-45, 12100-12250ft., Slide 3, K26/4, AGC 648. (Aptian).

Fig. 11. Cribroperidinium sp. A. A1-36, 7870-7880ft., Slide 3, K38/1, AGC 649. (Aptian-Albian). 
B. Thusu \& J. G. L. A. Van Der Eem

Plate 58

Aptian Dinoflagellate Cysts
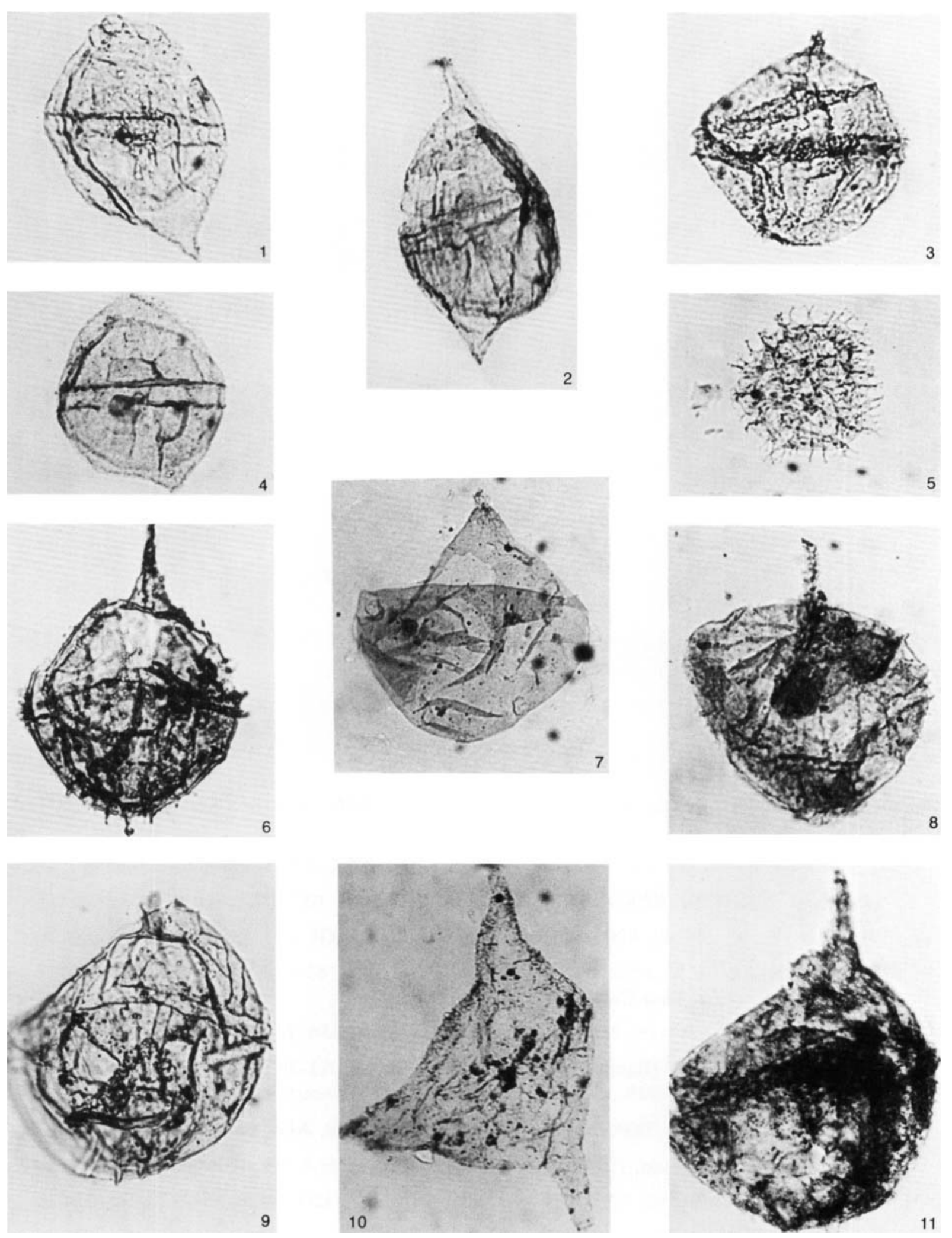


\section{Explanation of Plate 59}

All figures are $\times 500$

Fig. 1. Retitricolpites vulgaris Pierce, 1961. A1-NC 92, 6200-6210ft., Slide 4, P34/4, AGC 650. (AptianCenomanian).

Fig. 2. Psilatricolpites sp. A1-NC 92, 6200-6210ft., Slide 4, S35/0, AGC 651. (Aptian-Cenomanian).

Fig. 3. Eucomiidites sp. A1-NC 92, 6200-6210ft., Slide 4, M26/0, AGC 652. (Barremian-Cenomanian).

Fig. 4. Ephedripites sp. A. A1-NC 92, 6200-6210ft., Slide 4, W23/3, AGC 653. (Barremian-Cenomanian).

Figs. 5, 6. Afropollis sp. 5. A1-NC 92, 6200-6210ft., Slide 4, Y23/3, AGC 654. 6. A1-NC 92, 6200-6210ft., Slide 4, X25/3, AGC 655. (Aptian-earliest Cenomanian).

Fig. 7. Classopollis sp. ( 30 $\mu$ ) A1-28, $7613 \mathrm{ft}$., Slide 2, P33/3, AGC 656. (Aptian-Albian).

Figs. 8, 11. Callialasporites dampieri (Balme, 1957) Sukh Dev, 1961. 8. A1-NC 92, 6630-6640ft., Slide 3, T37/3, AGC 657. 11. B1a-18, 7300-7400ft., Slide 4, T45/0, AGC 658. (Neocomian-Aptian).

Fig. 9. cf. Trilites sp. A1-36, Core 8, 9600-9611 ft., Slide 1 unox., Y30/0, AGC 659. (Aptian-Albian).

Fig. 10. Reyrea polymorpha Herngreen, 1973. A1-36, 7870-7880ft., Slide 4, V35/2, AGC 660. (Aptian-Albian).

Fig. 12. Ephedripites sp. B. A1-NC 92, 6200-6210ft., Slide 4, X32/3-Y32/1, AGC 661. (Aptian-Albian).

Fig. 13. Collialasporites trilobatus (Balme, 1957) Sukh Dev, 1961. A1-NC 92, 6700-6710ft., Slide 4, T36/2, AGC 662. (Neocomian-Aptian). 
B. Thusu \& J. G. L. A. Van Der Eem Aptian Miospores
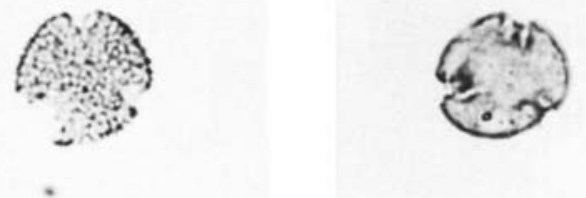

i)

2
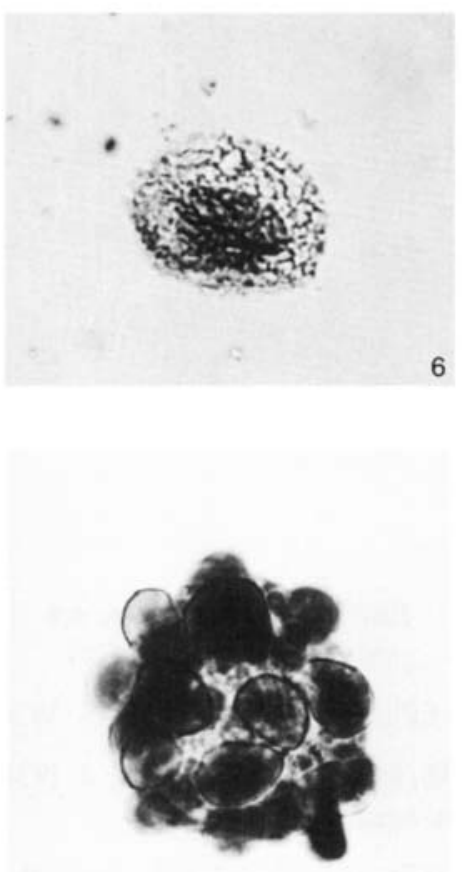

8

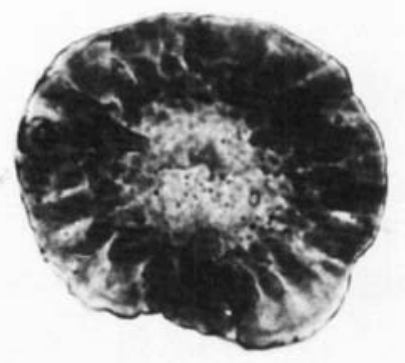

11

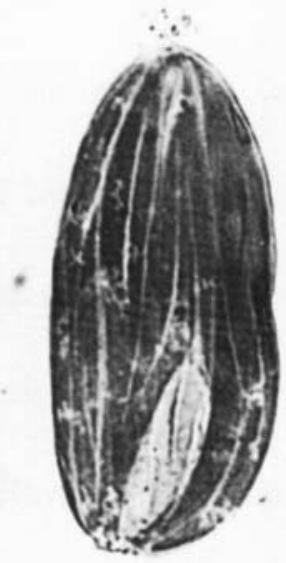

Plate 59
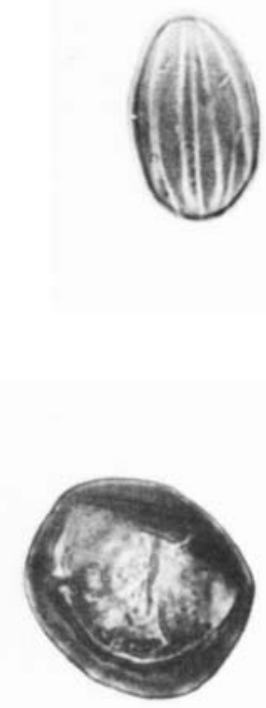

7

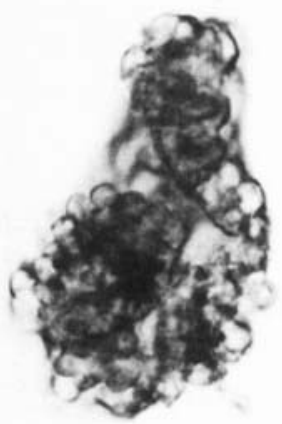

10

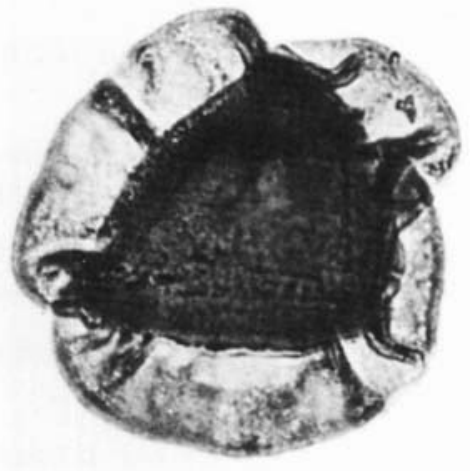

13 


\section{Explanation of Plate 60}

All figures are $\times 500$

Fig. 1. Tricolpites sp. A. A1-NC 92, 6200-6210ft., Slide 4, V30/3-W30/1, AGC 663. (Albian-Cenomanian).

Figs. 2, 3. cf. Nyssapollenites sp. 2. A1-NC 92, 6200-6210ft., Slide 4, N34/0, AGC 664. 3. A1-NC 92, 6200-6210ft., Slide 4, F29/0, AGC 665. (Albian-Cenomanian).

Fig. 4. Classopollis brasiliensis Herngreen, 1975. A1-35, 10300-10348ft., Slide 1, G34/1, AGC 666. (Cenomanian).

Fig. 5. Elaterocolpites castelainii Jardiné \& Magloire, 1965. G1-2, 7170-7210ft., Slide 2, G34/0-34/3, AGC 667. (Albian).

Fig. 6. Valensiella ovula (Deflandre, 1947) Eisenack, 1963. R3-82, 3416m., Slide 1, V32/0, AGC 668. (AlbianCenomanian).

Fig. 7. Elaterosporites klaszii (Jardiné \& Magloire, 1965) Jardiné, 1967. G1-2, 7170-7210ft., Slide 2, U26/0, AGC 669. (Albian).

Fig. 8. Xenascus ceratioides (Deflandre, 1937) Lentin \& Williams, 1973. B1-36, 10734-10738ft., Slide 3, U36/4, AGC 670. (Albian-Cenomanian).

Fig. 9. Pilosisporites trichopapillosus (Thiergart, 1949) Delcourt \& Sprumont, 1955. B1a-18, 6100-6200ft., Slide 4, T32/0, AGC 671. (Neocomian-Albian).

Fig. 10. Cyclonephelium vannophorum Davey, 1969. A1-NC 92, 6300-6310ft., Slide 5, L27/3, AGC 672 . (Neocomian-Cenomanian).

Fig. 11. Sofrepites legouxae Jardiné, 1967. (Contamination? Drilling Mud?) B1-36, Core 22, 10734-10738ft., Slide 2, U26/0, AGC 673. (Albian).

Fig. 12. Coronifera cf. oceanica Cookson \& Eisenack, 1958. E1-2, 5100-5200ft., Slide 5, V43/3, AGC 674. (Late Neocomian-Albian). 
B. Thusu \& J. G. L. A. Van Der Eem

Plate 60

Albian-Early Cenomanian Palynomorphs
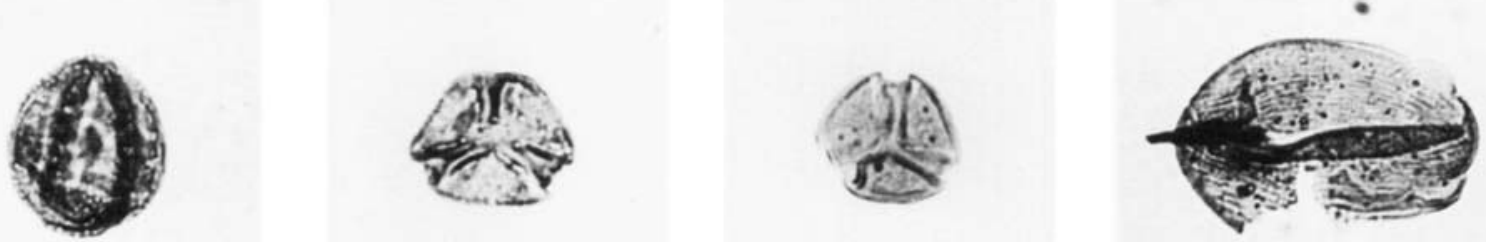

3
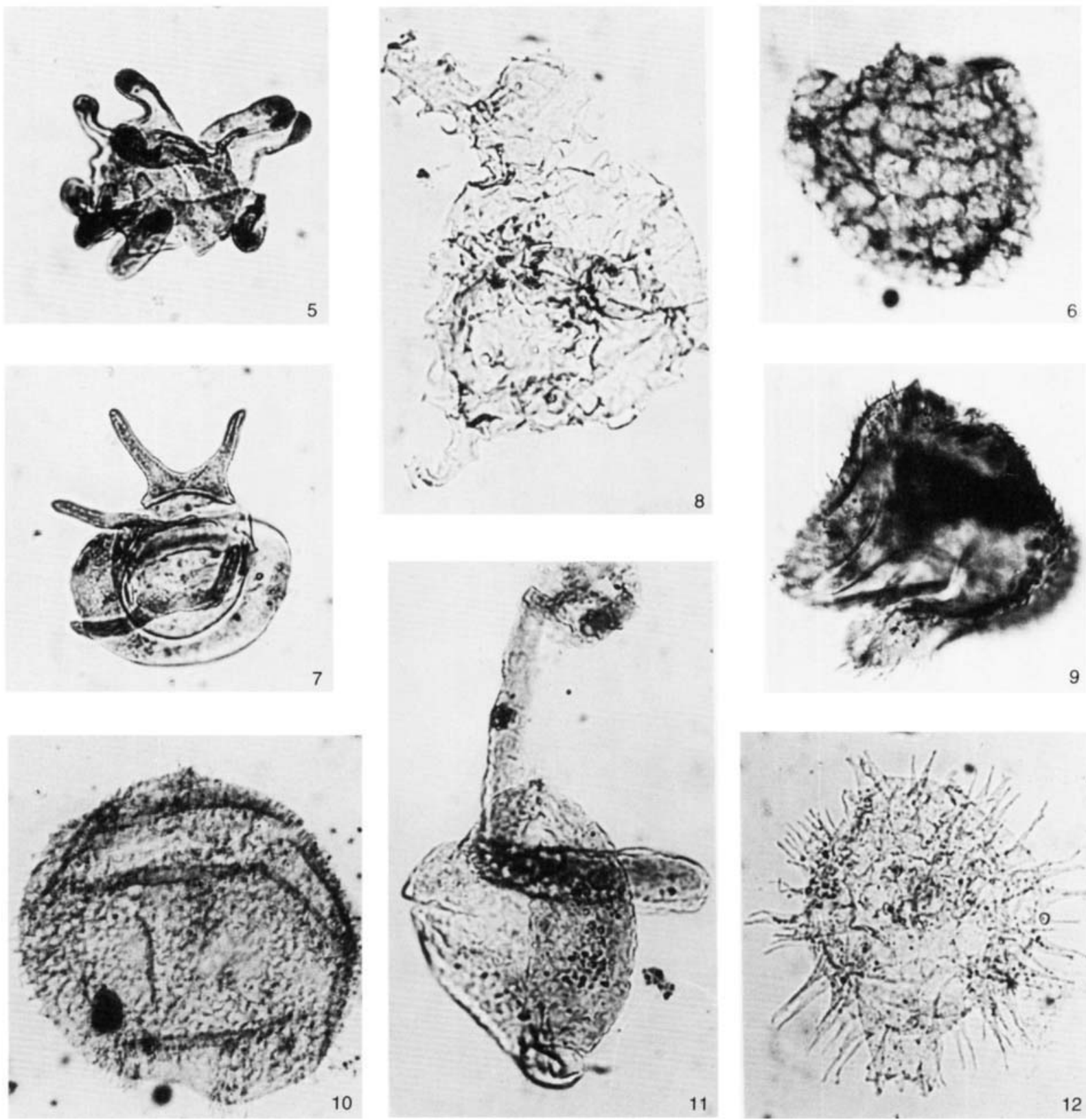